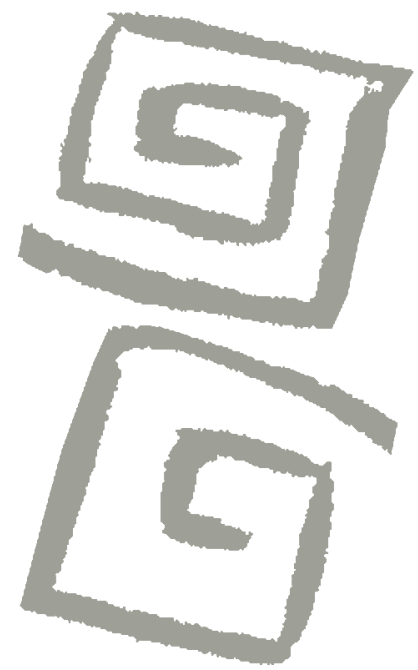

\title{
La salud colectiva en Brasil: analizando el proceso de institucionalización
}

\author{
Collective health in Brazil: analyzing the \\ institutionalization process
}

${ }^{1}$ Doctor en Ciencias. Profesor colaborador, Faculdade de Ciências Médicas, Universidade Estadual de Campinas (UNICAMP), São Paulo, Brasil. $\square$ (iD
RESUMEN En este trabajo analizamos la construcción de una tipología de estudios sobre la salud colectiva y las principales características del proceso de su institucionalización en Brasil, en el que se plantean como antecedentes tres momentos: el proyecto preventivo, la medicina social y la salud colectiva. Se abordan las etapas de institucionalización, disciplinarización y profesionalización de la salud colectiva en Brasil. Dentro de la etapa de institucionalización se analizan la conectividad y la comunicación; la regularización de los discursos y las prácticas; la construcción de una identidad y de las acciones políticas, y su incorporación y legitimación. Concluimos que la trayectoria de la construcción de la salud colectiva está marcada por tres dimensiones: la teórico-crítica, la político-sanitaria y la pedagógica-profesional.

PALABRAS CLAVES Salud Colectiva; Institucionalización; Brasil.

ABSTRACT This work first analyzes the construction of a typology of the studies on collective health and its institutionalization process in Brazil, in which three stages are proposed: the preventive project, social medicine and collective health. Secondly, the work examines the phases of institutionalization, disciplinarization and professionalization of collective health in Brazil. Within the institutionalization phase, the study analyzes connectivity and communication, regularization of discourses and practices, the construction of a separate identity and political actions, and the incorporation and legitimation of these elements. It is concluded that the trajectory of the construction of collective health is marked by three dimensions: the theoreticalcritical, the political-sanitary and the pedagogical-professional.

KEY WORDS Collective Health; Institutionalization; Brazil. 


\section{INTRODUCCIÓN}

No social group can plan for the future without a sense of who they are and what they do. ${ }^{(1 \mathrm{p} .276)}$

En 2015, dos eventos marcaron la presencia de la salud colectiva brasileña: los 35 años de la creación de la Asociação Brasileira de Saúde Coletiva (ABRASCO) y los 20 años de la revista Ciência \& Saúde Coletiva, órgano oficial de la asociación; dos momentos conmemorativos, que los historiadores podrán considerar relativamente recientes frente a las centenarias instituciones y publicaciones nacionales e internacionales en el campo de la medicina, la educación y la salud. Pero, como sabemos, si bien la salud colectiva tiene una historia reciente, su pasado tiene orígenes más distantes.

Participé de los dos eventos mencionados: por un lado, escribiendo el prefacio ${ }^{(2)}$ del libro sobre los 35 años de ABRASCO, organizado por Nísia Trindade Lima, Jose Paranaguá de Santana y Carlos Henrique Assunção Paiva y, por otro, elaborando un artículo para el número conmemorativo de los veinte años de Ciência \& Saúde Coletiva ${ }^{(3)}$ en el que se aborda el papel de esta revista en el proceso de institucionalización de la salud colectiva. Posteriormente, presenté algunas de esas ideas en el Congresso Brasileiro de Educação Médica ${ }^{(4)}$, en Río de Janeiro. Señalo estas referencias, pues en este artículo retomo y amplío las cuestiones formuladas en esos textos.

En este trabajo analizamos, en primer lugar, la construcción de una tipología de estudios sobre la salud colectiva y las principales características del proceso de su institucionalización en Brasil, en el que se plantean como antecedentes tres momentos: el proyecto preventivo, la medicina social y la salud colectiva. Luego se abordan las etapas de institucionalización, disciplinarización y profesionalización de la salud colectiva en Brasil y, dentro de la etapa de institucionalización, se analizan la conectividad y la comunicación, la regularización de los discursos y las prácticas, la construcción de una identidad y de las acciones políticas, y su incorporación y legitimación.

La construcción de una tipología de los estudios, así como de los abordajes utilizados en el estudio de la salud colectiva, fue fundamental para este trabajo, pues proveyó las bases documentales bibliográficas para el análisis del proceso de institucionalización de ese campo de conocimientos y prácticas.

\section{Hacia una tipología de estudios sobre la salud colectiva}

Cuando analizamos la historia y el concepto de salud colectiva constatamos que la forma de exponer y definir esta temática puede variar a partir de la fijación en la historicidad de los procesos que remiten a las formulaciones previas $^{(5)}$; a las cuestiones epistemológicas ${ }^{(6)}$; a los estudios sobre las áreas constitutivas del campo $^{(7)}$; a las formulaciones teóricas en las que se sustentan los abordajes de ese campo ${ }^{(8)}$ y del concepto de colectivo ${ }^{(9)}$; a las historias de vida que permean la construcción del campo $^{(10)[a] ;}$ a los análisis institucionales que refieren a la creación de espacios académicos específicos de actividades y carreras de grado ${ }^{(11)}$, de especialización ${ }^{(12)}$, de residencia ${ }^{(13)}$, de posgrado stricto sensu ${ }^{(14)}$, congresos $^{(15)}$, publicaciones ${ }^{(16)[b]}$; a los estudios que revisitan las construcciones curriculares en los diversos niveles de prácticas pedagógicas ${ }^{(17)}$; como también a los análisis que retoman la formación de recursos humanos ${ }^{(18)}$ y la inserción de la salud colectiva en la organización del sistema de salud ${ }^{(19)}$; las relaciones ciencia, tecnología y salud colectiva ${ }^{(20)}$, las relaciones salud colectiva y ciencia política ${ }^{(21)}$, las relaciones interdisciplinares ${ }^{(22)}$, y aquellas que toman a la propia ABRASCO como objeto de ponderación $^{(23,24)}$.

Por otro lado, es importante situar los diversos abordajes bajo los cuales ha sido analizada la salud colectiva. Ribeiro ${ }^{(25)}$ y $\operatorname{Luz}^{(8)}$ la consideraron como campo; García ${ }^{(26)}$, como un conjunto de prácticas teóricas, ideológicas, sociales, políticas, técnicas, que se aplicó al estudio sobre la educación médica 
en América Latina; Arouca $^{(27)}$ la inscribió en la investigación sobre el "dilema preventivista"; Nunes ${ }^{(28)}$, dentro de la medicina social latinoamericana, como institución que a lo largo de su historia pasa por diferentes etapas de institucionalización ${ }^{(2,3)}$; como construcción (constructo) producto de la interacción entre un pensamiento en salud (nivel filosófico/ ideológico), los conocimientos de diversos campos disciplinares (nivel cognoscitivo) y procesos organizativos (nivel político/social/ movimiento social) ${ }^{(3,29,30)}$ y y como espacio social, según Vieira da Silva. En este sentido, la salud colectiva es vista como el "producto del encuentro de un conjunto de agentes con trayectorias diferenciadas en lo que respecta a su recorrido, duración y posicionamiento en el campo científico, burocrático, político y de poder" $^{\prime \prime}(31$ p.41).

\section{Proceso de institucionalización: el concepto}

Tomando como referencia la institucionalización, es posible analizar la trayectoria de la salud colectiva como proceso histórico, político y sociológico. Para ello, buscamos en diversos estudios cómo ha sido entendido el proceso de institucionalización. Se destacan el sociólogo de la ciencia y de la educación superior Joseph Ben David (1920-1986), autor de estudios de referencia sobre la historia y las transformaciones de la ciencia moderna, contextualizados en sus espacios institucionales, políticos y culturales ${ }^{(31)}$; Anthony Oberschall (1936), físico y doctor en sociología, con sus análisis sobre la historia de la institucionalización de las disciplinas y la sociología en EE.UU. ${ }^{(32)}$; el sociólogo de la medicina Samuel W. Bloom (1921-2006) con la trayectoria de la sociología médica en EE.UU.(33); la socióloga australiana Fran Collyer, aplicando el concepto de institucionalización al estudio de la sociología de la medicina/salud en EE.UU., Reino Unido y Australia ${ }^{(1)}$. Para esos autores, las etapas de ese proceso serían cuatro, las cuales sintetizamos a continuación.
Primera etapa: pequeños grupos -generalmente colegas de trabajo, profesores de un determinado departamento de la universidad o investigadores- se reúnen informalmente e inician la discusión de temas, métodos y técnicas de conocimiento de las disciplinas, entrando en contacto con las experiencias desarrolladas en otros lugares, o con expertos de otros países o instituciones y, como describe Collyer, con el propósito de "enfocar un conjunto de problemas o experiencias mutuas o marginales, más que temas teóricos per se" (1 p.52).

Segunda etapa: la disciplina anteriormente considerada periférica pasa a ser vista como parte de un determinado campo de conocimientos y se torna significativa en el plano de la cultura, hay mayor regularidad en las reuniones y debates y se desarrollan estrategias para atraer a nuevos miembros. Como señalan los estudiosos del proceso de institucionalización, en este momento se produce "la regularización de los discursos, prácticas

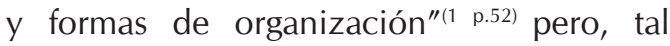
como mencionan Oberschall y Ben-David, "la disciplina emergente necesita conseguir un grupo patrocinador que la apoye con recursos financieros" ${ }^{\prime \prime 3}$ p.42).

Tercera etapa: el nuevo campo pasa a ser significativo para un área en su conjunto y se comienza a estandarizar la forma de reclutar recursos humanos y financieros, $y$ de seleccionar especialistas. Se trata de la construcción de una "identidad distinta" y de "acciones políticas" que se desarrollan dentro y fuera de la academia; se discute la "naturaleza de la disciplina, su estatus actual y probabilidades futuras"(1 p.52). Bloom ${ }^{(33}$ p.44) destaca que, en esta fase, como en la anterior, por ejemplo, "las fundaciones fueron una fuerza vital" en la institucionalización de la sociología en EE.UU.

Cuarta etapa: se produce la consolidación del nuevo campo o disciplina, construyendo su cultura dentro de la comunidad científica, con sus redes sociales de comunicación, asociaciones científicas y publicaciones propias; se trata de un momento en el cual la disciplina establece distintos programas, y formas de acreditación de "considerable autonomía" 
por parte de los miembros senior de la disciplina en sus respectivos departamentos ${ }^{(1) .53)}$.

\section{Salud colectiva: etapas de la institucionalización}

Las referencias a la publicación de la revista Ciência \& Saúde Coletiva y a los 35 años de ABRASCO, conmemorados en el $11^{\circ}$ Congreso Brasileño de Salud Colectiva realizado en Goiânia, en 2015, marcan claramente el cuarto momento de la institucionalización de un campo de conocimiento. Esos dos acontecimientos forman parte de las realizaciones que dan visibilidad al campo, extendiéndolo más allá de las fronteras exclusivas de los especialistas. En realidad, este cuarto momento cobra sentido al retomar los antecedentes de la salud colectiva.

En trabajos anteriores, señalamos la higiene, la medicina preventiva y social, la medicina integral, la medicina comunitaria y la salud pública convencional como antecedentes, y los demarcamos en determinadas etapas, aclarando que se trata de trabajar con recortes en el tiempo y no con una historia lineal. La palabra etapas - utilizada aquí- se refiere a esa percepción de la trayectoria histórico-conceptual de la salud colectiva como un proceso.

Desde una perspectiva temporal (de 1950 hasta la actualidad), en este trabajo analizamos las tres etapas que emergen al transitar la historia de la salud colectiva, vista globalmente: el proyecto preventivista, la medicina social y la salud colectiva.

\section{El proyecto preventivista}

De forma genérica, denominamos proyecto preventivista a los antecedentes que se ubican entre 1950 y 1970 . Ese proyecto privilegió las prácticas pedagógicas transmitidas por la Organización Panamericana de la Salud (OPS) en seminarios que se impartieron en la década de 1950 en diversas ciudades: Colorado Springs (1952), Nancy (1952), Gotemburgo (1953), Viña Del Mar (1955), Tehuacán (1956). Ante la inexistencia de una teoría que fundamentara esas prácticas, se extendió el uso del "modelo de la historia natural de la enfermedad", creado por Hugh R. Leavell y Edwin G. Clark ${ }^{(34)}$. Años más tarde, los niveles de prevención fueron reelaborados por García ${ }^{(35)}$ con el objetivo de incorporarlos en la enseñanza de las ciencias de la conducta a los estudiantes de medicina. La medicina preventiva es un movimiento ideológico originado en EE.UU., que se expandió por América Latina, buscando reorientar la práctica médica hacia una actitud integral, preventiva, comunitaria y social de los estudiantes de medicina, reestructurando el $\mathrm{CO}^{-}$ nocimiento médico.

Este fue un período de intensa actividad docente en los departamentos de medicina preventiva y social que se crearon en las escuelas médicas brasileñas. Los departamentos más antiguos son el de la Faculdade de Medicina de Ribeirão Preto de la Universidade de São Paulo (USP) de 1954, y el de la Universidade Federal de Minas Gerais (UFMG) de 1958. Una gran parte de los departamentos se crearon en la década de 1960 y en los inicios de la década de 1970: en 1965, en la Faculdade de Ciências Médicas da Santa Casa de São Paulo y en la Faculdade de Ciências Médicas de la Universidade Estadual de Campinas (UNICAMP); en 1967, en la Faculdade de Medicina de la USP; y en 1970, en la Universidade Federal do Rio de Janeiro (UFRJ), y la Universidade Federal da Bahia (UFBA).

En ese contexto, las prácticas académicas (basadas en la prevención y la educación sanitaria), incluyendo las primeras experiencias de actividades de enseñanza conocidas como actividades extramuros (trabajos en barrios y comunidades urbanas periféricas) sufren críticas y, en la segunda mitad de los años 1970, se acentúan las rupturas que ya se hacían sentir al inicio de la década. Un ejemplo de esto es el impacto que produjeron en 1972, por un lado, la publicación del estudio de García ${ }^{(26)}$ sobre la educación médica en América Latina, que enmarca el tema dentro del estructuralismo marxista y, por otro, la realización de la Reunión sobre Enseñanza de las Ciencias Sociales en las 
Facultades de Ciencias de la Salud (Cuenca, Ecuador $^{(36)}$, que critica profundamente al funcionalismo sociológico. Como se señala en diversos estudios, en paralelo a la crisis epistemológica en el campo de la medicina preventiva y social, había una crisis generalizada de los servicios de salud que, en el caso brasileño, se sumaban a las transformaciones del sistema de la seguridad social. Todo esto sucedía en un contexto político caracterizado por el autoritarismo, la represión política, la censura a la prensa, la persecución a los docentes y la detención de militantes. Recordemos que la dictadura militar en Brasil se extiende desde el golpe de Estado de 1964 hasta 1985, con la elección indirecta a través del Colegio Electoral de Tancredo Neves, por entonces ex gobernador de Minas Gerais, que muere antes de ocupar su cargo, y asume la presidencia de la República el vicepresidente José Sarney.

Desde el punto de vista de los principales fundamentos teórico-epistemológicos, la etapa preventivista aborda no solo la salud como producción social -mostrando los límites de los modelos centrados en la biomedicina- sino también la inclusión en la enseñanza de disciplinas y temas que traducen las relaciones salud-sociedad. Si en los años pioneros de la medicina preventiva, la creencia era que las transformaciones profesionales y del sistema asistencial se fortalecerían con los cambios curriculares en la enseñanza de grado, en los años 1970 se inicia la programación de la formación de posgrado. Así, podemos decir que en ese período se produce una intensa problematización de la salud versus la medicina, estableciendo lo que los estudiosos de la sociología del conocimiento denominan conectividad y comunicación, que abre otra etapa en el proceso de institucionalización: la "regularización de los discursos, prácticas y formas de organización"(1).

En ese movimiento emergen manifestaciones académicas ${ }^{(3)}$, pioneras en el estudio de la salud-enfermedad-instituciones de salud: el estigma de la lepra de Gandra ${ }^{(37)}$; la crítica a la medicina preventiva de Arouca ${ }^{(27)}$; la práctica médica en la ciudad de São Paulo y el análisis de la medicina comunitaria de Donnangelo ${ }^{(38,39)}$; el estudio del "discurso arqueológico" de la medicina social brasileña de Machado et al. ${ }^{(40)}$; las instituciones médicas de Luz $^{(41)}$; el consumo de medicamentos de Cordeiro ${ }^{(42)}$; la prevención como proceso político de $\mathrm{Cohn}^{(43)}$; las relaciones entre educación médica y capitalismo de Schraiber ${ }^{(44)}$; las relaciones de trabajo y la prevención social de Marsiglia ${ }^{(45)}$; los estudios sobre seguridad social y políticas de salud de Teixeira ${ }^{(46)}$; el análisis teórico de las raíces sociales del trabajo médico de Mendes-Gonçalves ${ }^{(47)}$; el análisis crítico de la medicina comunitaria de Paim ${ }^{(48)}$; la "cuestión de la salud" en Brasil de Braga ${ }^{(49)}$; los estudios epidemiológicos sobre nutrición y desnutrición, la relación entre la enfermedad de Chagas y la estructura agraria, el trabajo urbano, las transformaciones del espacio agrario ${ }^{(50,51,52)}$.

En 1981, al hacer una revisión de la producción científica de los años 1970, Donnangelo recordaba que esa producción en medicina preventiva, medicina social y salud colectiva no era uniforme y reflejaba
...la amplitud de las connotaciones asumidas por la noción de colectivo: colectivo/medio; colectivo/conjunto de individuos; colectivo/interacción entre elementos; colectivo como conjunto de efectos o consecuencias de la vida social; colectivo transformado en social como campo específico y estructurado de prácticas. ${ }^{(8 p .27)}$

Aunque nuestro abordaje se centre en Brasil, no podemos dejar de destacar la relevancia que tuvieron estos autores sobre el pensamiento social en salud de otros países de América Latina desde la década de 1970: Breilh ${ }^{(53)}$, en la conceptualización de la reproducción social y la determinación social de la salud; Laurell(54), sobre la colectividad como expresión del proceso salud-enfermedad; y Juan César García, María Isabel Rodríguez y Miguel Márquez, por el empuje que le dieron a la medicina social ${ }^{(55)}$. 


\section{La medicina social}

En un escenario fuertemente impregnado por la búsqueda de innovaciones y alternativas, al romper con los modelos convencionales de la medicina preventiva y social, se transita hacia un "nuevo" proyecto, la medicina social, que tiene sus raíces más distantes en los movimientos europeos de la medicina social de 1848. Según Nunes, en Brasil, "la emergencia de esos proyectos refleja, de un modo general, el contexto socioeconómico y político-ideológico más amplio, como también las sucesivas crisis, presentes tanto en el plan epistemológico, como en las prácticas de salud y en la formación de recursos humanos" ${ }^{\prime \prime}$ p.2). Esas ideas permean las reuniones realizadas, desde 1968 hasta 1973, por los docentes de los Departamentos de Medicina Preventiva y Social del estado de São Paulo, al discutir, entre otros temas, las corrientes de pensamiento entonces existentes: la medicina preventiva y la medicina social; la enseñanza disciplinaria, especialmente, de las ciencias sociales y la epidemiología; los trabajos extramuros y las relaciones con los servicios de salud.

La reconquista de las discusiones sobre la medicina social también forma parte del documento de 1974 de la Organización Panamericana de la Salud (OPS), momento en que ese organismo asume que el objeto de la medicina social debe ser entendido como:

...el campo de la práctica y los conocimientos relacionados con la salud. Teniendo este objetivo como su preocupación central, la medicina social trata de estudiar la sociedad, de analizar las formas corrientes de interpretación de los problemas de salud y de la práctica médica. ${ }^{(56 \text { p.5) }}$

Reconoce los límites de los modelos anteriormente transmitidos y al retomar la discusión sobre la enseñanza establece que:

...en vez de añadir nuevos contenidos al campo imprecisamente delimitado de la medicina preventiva, sería preferible la delimitación de este campo dentro de una perspectiva más realista de las posibilidades de enseñanza de la medicina y de las limitaciones en su eficacia como determinante de la forma en que las prácticas de la atención médica y de la salud asumen en un momento determinado en cada formación social concreta. ${ }^{(56 \text { p. 4) }}$

De modo general, el marco conceptual que suministraba las bases para recapacitar ese campo de prácticas consideraba que:

En cada formación social concreta la educación médica cumple un papel fundamental en la reproducción de la organización de los servicios de salud y se cristaliza en la reactualización y preservación de las prácticas específicas, tanto en las dimensiones del conocimiento cuanto en las técnicas y contenido ideológico. Además de esto, es la propia estructura de la atención médica la que ejerce una acción dominante sobre el proceso de formación de recursos humanos, principalmente a través de la estructura del mercado de trabajo y de las condiciones que circunscriben la práctica médica. ${ }^{(56 \text { p.3 }}$

Esa forma mecánica de determinación de la práctica sobre la enseñanza no pasó desapercibida para algunos estudiosos, que apuntaron la necesidad de que la universidad preservara algún grado de autonomía ${ }^{(57)}$.

En esta segunda fase del proceso de institucionalización, constatamos la presencia de asignaturas de las ciencias sociales (sociología, antropología y ciencia política) y de la epidemiología social, y la formalización de las primeras carreras de posgrado en medicina social, en una vertiente diferente de la anteriormente existente en los cursos de salud pública convencionales.

En Brasil, el primer curso stricto sensu de maestría en medicina social fue creado en 1973, junto al Instituto de Medicina Social, mientras que los cursos stricto sensu, tanto de maestría como de doctorado, en salud pública, medicina preventiva o medicina 
comunitaria, surgen unos años antes. En la USP, en 1970, se crean posgrados en la Faculdade de Saúde Pública; en 1971, en la Faculdade de Medicina de Ribeirão Preto; y, en 1973, en la Faculdade de Medicina de São Paulo. En la UFBA, en 1973, se crea la Maestría en Salud Comunitaria en la Faculdade de Medicina. Un antecedente de estos cursos fue la creación, en 1967, de la Maestría en Salud Pública de la Escola Nacional de Saúde Pública (ENSP), la cual se dictó durante tres años hasta 1969, cuando fue cerrada, y luego se reabrió en 1977.

Otros elementos que se añaden a esta segunda fase de institucionalización se refieren al papel de instituciones como la OPS, y la presencia de pensadores europeos que trajeron importantes contribuciones al pensamiento social en salud. Por ejemplo, en 1974, llega a Brasil, por invitación del Instituto de Medicina Social de la UERJ, el filósofo francés Michel Foucault (1926-1984), quien presenta las emblemáticas conferencias sobre el nacimiento de la medicina social y el nacimiento del hospital. En 1978, el médico Giovanni Berlinguer (1924-2015), invitado por el Centro Brasileiro de Estudos da Saúde (CEBES), realiza exposiciones, reuniones, debates y lanza la traducción de su libro Medicina y política, que influyó fuertemente en el movimiento de la Reforma Sanitaria Brasileña ${ }^{(58)}$.

En 1976, en el momento en que se acentuaban las críticas al proyecto preventivo, se analizaba teórica y críticamente otro movimiento ideológico -la medicina comunitariaque traía desde sus orígenes el ideario de la medicina integral, los abordajes sociológicos sobre la comunidad y el servicio social, procedentes de los años 1960. Paim señala sus limitaciones al mencionar que "sus proposiciones no le exigían modificaciones relevantes a la organización del sistema de atención médica, especialmente, con relación a las contradicciones entre los subsistemas de producción de los servicios públicos y privados"(48 p.11). Estas críticas coinciden con las discusiones que examinan la planificación en salud en América Latina y la sitúan, ya no desde la perspectiva economicista (CENDES/OPS) anteriormente adoptada, sino como "posibilidad política”"(59 p.37)

Con relación a los movimientos organizativos, en 1976 se crea el CEBES, que tiene como uno de los ejes de sus actividades la lucha por la democratización de la salud y la sociedad ${ }^{(58)}$. En el plano internacional, la Conferencia Internacional sobre Atención Primaria de Salud, realizada del 6 al 12 de septiembre de 1978, formula la Declaración de Alma-Ata. También en 1978 se realiza el $1^{\circ}$ Encuentro Nacional de Posgrados en Salud Colectiva, en Bahia, donde se discute la formación de una asociación de posgrado y, al año siguiente, en octubre de 1979, se realiza el $1^{\circ}$ Simposio sobre Política Nacional de Salud en la Cámara Federal, donde el CEBES presenta el documento titulado $A$ questão democrática na área da saúde ${ }^{(60)}$, que se tornó un texto de referencia en el proceso de la Reforma Sanitaria. En un año cargado de realizaciones ocurre la reglamentación de la Residencia en Medicina Preventiva y Social y el inicio de la Residencia en Medicina Social asociada al Instituto Nacional de Asistencia Médica de la Seguridad Social (INAMPS), creado en 1974 y cerrado en 1993.

\section{La salud colectiva}

Muchos aspectos señalan que están dadas las condiciones objetivas para una nueva fase de la salud colectiva que, siguiendo el proceso de institucionalización, corresponde a la etapa de incorporación. En este momento, la identidad del campo se construye en un proceso de formación sistemática, no solo con relación a su propia estructura interna (formulación epistémica), sino también con estructuras y organizaciones externas al campo (educacionales, gubernamentales) burocratizando y legalizando el campo. A esa incorporación se asocia el papel de la Financiadora de Estudos y Projetos (FINEP) que da apoyo financiero a 87 proyectos de investigación en el período 1968-1979, y del Conselho Nacional de Desenvolvimento Científico e Tecnológico (CNPq), que concedió, de 1976-1980, las primeras 20 becas a investigadores en salud pública, de un total 
de 113 becas, de las cuales 67 fueron para el área clínica y 26 para nutrición. También se amplía el proceso de divulgación (revistas científicas, libros, producción técnica y periodística) y comienzan a hacerse con regularidad reuniones, seminarios y congresos liderados por comités de especialistas.

Las experiencias acumuladas a lo largo de la década de 1970 culminan en 1979 con la creación de la Associação Brasileira de Posgraduação em Saúde Coletiva, más tarde denominada Associação Brasileira de Saúde Coletiva (ABRASCO).

La creación de ABRASCO se concreta en uno de los momentos histórico-políticos de mayor importancia para Brasil. En 1979, a través del Colegio Electoral, el Congreso formaliza al General João Baptista de Oliveira Figueiredo (1918-1999) como el $5^{\circ}$ presidente de la dictadura, para que gobierne hasta 1985; en este periodo se revoca el Acto Institucional No 5 (Al-5) emitido por la dictadura, se deroga la censura previa, se restaura el habeas corpus y, entre otras medidas, se sanciona la Ley de la Amnistía y los exiliados comienzan a regresar al país (cerca de 2.500 personas), se restaura el pluripartidismo y surgen nuevos partidos ${ }^{(61)}$.

Por lo tanto, podría decirse que en 1979 se inicia la cuarta etapa del proceso de institucionalización de la salud colectiva: la de la legitimación y con ella las medidas de inclusión de nuevos actores, programas, revistas científicas, etc.

En la salud colectiva se repite el mismo fenómeno que se producía en otros campos del conocimiento, incluyendo la sociología y sus subespecialidades, como la sociología médica o la sociología de la salud. Según Collyer $^{(1)}$ p.53), en la etapa de legitimación, las nuevas disciplinas legalizan, formalizan, y establecen su representación en consejos consultivos y comités universitarios.

Además, la legitimación puede incluso oficializarse en documentos que trazan objetivos y programas al momento de la creación de la nueva disciplina, campo de prácticas, o de una asociación. En el caso de ABRASCO, verificamos que el documento preliminar de su creación, en 1979, afirma:
En términos de contenido programático, la Asociación reconoce que se deba establecer un adecuado equilibrio entre los contenidos técnicos y teórico-conceptuales, entre lo "biológico" y lo "social", entre lo "operacional" y lo "crítico", como forma de evitar el "tecnicismo" y el "biologismo" presentes en la tradición de enseñanza del área de salud colectiva. (62 p.114) (cursivas añadidas)

La creación de foros específicos se produciría en las décadas siguientes: el Fórum de Coordenadores dos Cursos de Pós-Graduação em Saúde Coletiva (1994), el Fórum de Graduação em Saúde Coletiva y el Fórum de Editores de Saúde Coletiva (2014). Estos espacios permiten no solo la discusión actualizada de los problemas de sus áreas de actuación, sino también establecer puentes con otras asociaciones, órganos gubernamentales y asociaciones internacionales.

En ese proceso de legitimación, la creación de una revista, como portavoz del campo de la salud colectiva, se concreta en 1996 con la publicación en forma regular de Ciência \& Saúde Coletiva. Hasta esa fecha, ABRASCO tenía como medios de divulgación el Boletim da ABRASCO, editado desde 1982, y una serie inicialmente denominada Ensino da saúde pública, medicina preventiva e social no Brasil (publicó tres números desde 1982) y posteriormente Estudos de Saúde Coletiva (publicó dos números en 1986 y 1988), que dejaron de publicarse.

Según Ferreira ${ }^{(63)}$ el periodismo médico en Brasil surge en la primera mitad del siglo $X I X, y$ entre la segunda mitad de ese siglo y segunda década del siglo XX aparecen una serie de revistas como: Gazeta Médica do Rio de Janeiro (1862), Gazeta Médica da Bahia (1866), Memórias do Instituto Oswaldo Cruz (1909) y los Anais da Academia Brasileira de Ciências (1917). Recién a fines de la década de 1960 y comienzos de la siguiente aparecen nuevas revistas: en 1967, la Revista de Saúde Pública de la Faculdade de Saúde Pública de la USP; en 1970, la Revista Brasileira de Saúde Ocupacional de Fundacentro; en 
1974, la Revista Baiana de Saúde Pública y, en 1976, Saúde em Debate del CEBES.

En las décadas de 1980 y 1990 surgen nuevas publicaciones: en 1985, Cadernos de Saúde Pública de la Escola Nacional de Saúde Pública; en 1987, Cadernos de Saúde Coletiva del Instituto de Estudos em Saúde Coletiva de la UFRJ; en 1991, Physis - Revista de Saúde Coletiva del Instituto de Medicina Social de la UERJ; en 1992, Saúde e Sociedade de la Faculdade de Saúde Pública de la USP, en colaboración con la Associação Paulista de Saúde Pública (APSP); en 1994, História, Ciências, Saúde - Manguinhos de la Casa de Oswaldo Cruz, Fundação Oswaldo Cruz; en 1997, Interface - Comunicação, Saúde, Educação de la Universidade Estadual Paulista (campus Botucatu); y, en 1998, la Revista Brasileira de Epidemiología de ABRASCO.

Todas estas publicaciones han sido de fundamental importancia para el campo ${ }^{(16)}$, sin embargo, Ciência \& Saúde Coletiva es un hito en la consolidación del proceso de institucionalización del campo de la salud colectiva. Una de sus características es tener un eje temático. Minayo, Gomes, Almeida, Goldbaum y Carvalheiro ${ }^{(64)}$ señalan que, en el período 1996-2014, la diversidad temática del material publicado llega a un total de 112 temas: políticas de salud $(13 \%)$, salud del trabajador $(7 \%)$, salud y ambiente $(7 \%)$, salud del niño y del adolescente $(6 \%)$, información y salud $(6 \%)$, ciencia y salud colectiva $(6 \%)$, salud de los ancianos (5\%), ciencias sociales y humanas en salud (5\%), violencia como cuestión de salud pública (5\%) y, en porcentajes menores, género y salud, alimentación, adicción a las drogas, planificación en salud, tecnología y salud, cuestiones sociales y salud, formación en salud, calidad de vida, evaluación en salud bucal, y aspectos epistemológicos de la epidemiología. En ese mismo artículo, los autores analizan otra revista de ABRASCO, la Revista Brasileira de Epidemiología, fundada en 1989, cuyas principales temáticas abordadas de 2012 a 2014 fueron: enfermedades infecciosas y crónicas, nutrición y estudios metodológicos, que abarcaron el $52 \%$ del total de temas. Mencionan que, además, se abordaron otras temáticas como violencia y salud, estilo de vida/comportamiento, salud del anciano, salud de la mujer y de la infancia y salud bucal.

No es nuestro objetivo detallar la extensa producción en salud colectiva, pero cabe recordar que en la última fase de la institucionalización del campo se publica, en 2006, el primer Tratado de Saúde Coletiva, que fue reeditado en $2012^{(65)}$. En sus casi 800 páginas identifica el campo y su principales temas, suministra fuentes seguras, formalizadas y sistematizadas, para los profesores, estudiantes, investigadores y profesionales de salud. En 2014, se publicó un segundo tratado titulado Saúde Coletiva - teoria e prática ${ }^{(66)}$. Sin duda, la publicación de un tratado (tractatus) garantiza todo un proceso de constitución, consolidación, legitimación y sistematización de ese campo de conocimiento.

Como buscamos mostrar, no se puede entender el proceso de institucionalización, sin la presencia de los otros dos procesos complementarios: la disciplinarización y la profesionalización.

\section{La disciplinarización}

Acompañando la trayectoria de la salud colectiva, la disciplinarización lato sensu del campo ya venía ocurriendo desde la década de 1970 cuando las carreras de posgrado iniciaron la formalización de sus estructuras académicas, la cual se completó al conformarse la gran área de "ciencias de la salud", en la Coordenação de Aperfeiçoamento de Pessoal de Nível Superior (CAPES). Dentro de esa área, en la actualidad hay 77 programas, de los cuales 45 son académicos: 13 maestrías, 3 doctorados, y 29 maestrías y doctorados desarrollados en forma conjunta. Recién veinte años después, en 1990, se crean los cursos de posgrado profesionales (distintos de los académicos) que, en la actualidad, Ilegan a un total de 32 cursos de maestría en salud colectiva. El proceso de disciplinarización se completó con la formación de grado en salud colectiva que, en 2014, ya contaba con 21 cursos implementados $^{(11 \text { p. 147). }}$. 
Incluimos esta información preliminar en el proceso de disciplinarización, la cual será retomada al abordar la profesionalización, pues entendemos que ambos procesos se complementan, tal como lo señalara Collyer: "la reciente renovación del interés en las disciplinas [y del proceso de disciplinarización] ha socavado la visión de estas como dominios esencialmente cognoscitivos" ${ }^{\prime \prime 1 ~ p .38)}$ o sea, son "formas sociales producidas a través de procesos sociales"(1 p. 256). Estos puntos son importantes porque, según Collyer, las disciplinas pueden ser vistas "como el locus de la acción social y como instituciones que estructuran y regulan esa acción" (1 p. 14).

\section{La profesionalización}

Las observaciones desarrolladas en las líneas anteriores nos Ilevan a considerar el otro proceso, el de la profesionalización, como parte integrante del proceso de institucionalización, que se desarrolla desde la formación escolar (diploma) y se extiende al mandato que, según el clásico texto de Hughes, es lo que la sociedad define como "conducta apropiada con respecto a los temas pertinentes en su trabajo" a partir de un sentido común de "autoconciencia y solidaridad" (67 p.78).

El profesionalismo aún deberá formalizar organizaciones que controlen y protejan el ejercicio profesional.

En el campo de la salud pública o salud colectiva, la fase inicial de la formación de profesionales especialistas tuvo lugar en las facultades de salud pública, tanto de médicos, como de los primeros científicos sociales y educadores en salud y enfermeras en salud pública, siendo que la Ley 488 , de 1948, ya menciona médicos sanitaristas y la Ley 3427 de 1958 ingenieros sanitaristas. Posteriormente, la complejidad resultante de la inter y de la multidisciplinaridad, y las necesidades pautadas por la Reforma Sanitaria y por los servicios de salud, pasaron a exigir una creciente profesionalización de sus cuadros de trabajo frente a las nuevas demandas de la salud colectiva en planificación y gestión, vigilancia sanitaria, vigilancia ambiental, ciencias sociales en salud y salud del trabajador, entre otras.

Sin duda, desde 1970 el área cumplía la función de formar cuadros académicos e investigadores y, en esta última categoría, los datos de 2010 del CNPq muestran que de un total, para todas las áreas de conocimiento, de 162.295 investigadores brasileños y 4.477 investigadores extranjeros, correspondían al área de salud colectiva 5.518 brasileños y 123 extranjeros, distribuidos en 732 grupos de investigación $(2,7 \%$, sobre un total de 27.523 grupos en todas las áreas de conocimiento); los cuales, para 2014, sumaban 975 grupos $(2,8 \%$, sobre un total de 35.424 grupos en todas las áreas de conocimiento), con 8.003 investigadores, de los cuales 159 eran extranjeros ${ }^{(68)}$. Sin embargo, las carreras de grado de sanitaristas en salud colectiva, y la formación de posgrado profesional, como ya mencionamos, forman parte de la historia más reciente de la salud colectiva en Brasil.

Las carreras de grado en salud colectiva se discutían desde fines de la década de 1970, pero la propuesta preliminar de esa idea recién se concretó mucho más tarde, en 2001, tanto en la UFBA, como en la Universidade Estadual do Rio Grande do Sul (UERGS) (con la carrera de Administración de sistemas y servicios de salud) y en la UFRJ, con la creación de la carrera de grado en salud colectiva. En 2002, la UFBA organizó un amplio debate sobre la cuestión analizando la pertinencia de la formación de un nuevo profesional en el ámbito de las carreras de grado. Cronológicamente, el tema de la formación de grado en salud colectiva se retoma en 2010, cuando se crea el Foro de Graduación en Salud Colectiva ${ }^{(11)}$.

En 1995, se reglamentan las maestrías profesionales y, en el área de salud colectiva, los primeros programas los ofrecieron instituciones que "ya tenían programas de posgrado afirmados y de óptima calidad; el Instituto de Medicina Social de la UERJ, el Instituto de Salud Colectiva de la UFBA y la ENSP de Fiocruz" ${ }^{\prime 69}$ p.203). Según Cesse e Veras ${ }^{69}$ p.204), "en la actualidad las maestrías profesionales tienen reconocimiento en la comunidad científica". También muestran que el 
“mayor número de maestrías profesionales se da en las áreas interdisciplinarias, enseñanza, administración y salud colectiva". En términos proporcionales, la salud colectiva es el área con el segundo mayor porcentaje de maestrías profesionales, con el $41,6 \%$ de las carreras ofrecidas bajo esta modalidad, mientras que el área de educación se ubica en el primer lugar con el $57,3 \%$ de maestrías profesionales. Estos autores señalan que hay problemas y desafíos en esa área de formación tanto desde el punto de vista pedagógico como en el perfeccionamiento de los mecanismos de evaluación y financiación, siendo indiscutible su presencia en la formación de recursos estratégicos e innovadores para la salud colectiva.

\section{CONSIDERACIONES FINALES}

Al establecer nuestras consideraciones en el proceso de institucionalización, disciplinarización y profesionalización se evidencia que la trayectoria de la construcción de la salud colectiva está marcada por tres dimensiones: la teórica-crítica, la político-sanitaria y la pedagógica-profesional. Pensamos que esas tres dimensiones ofrecen un marco de referencia que puede ser la fuente de las nuevas demandas, tanto vinculadas a las innovaciones tecnológicas e informacionales, como a las políticas sociales -en sentido amplio- y a las políticas de salud, como foco directo de atención, incluyendo las cuestiones ambientales y las del mundo del trabajo.

Consideramos que el proceso de institucionalización es un proceso dialéctico que se consuma como un movimiento histórico a través de acuerdos en los que las instituciones de investigación, de enseñanza, y los servicios de salud se vuelcan a la búsqueda, formación y rotación de investigadores, profesores y gestores, materializados en la producción de servicios de salud y producción intelectual bajo las más diversas formas (libros, artículos, películas, notas periodísticas, etc.).

En ese sentido, en este trabajo buscamos mostrar que la salud colectiva se encuentra institucionalizada y legitimada pero abierta a nuevas posibilidades de transformación y recreación de sus espacios de actuación. Como escribe $\mathrm{Luz}^{(8)}$, en la actualidad la salud colectiva se constituye como "una estructura discursiva semiabierta, con inclusión continua de disciplinas oriundas de diferentes campos científicos", que se incorporaron a lo largo de su historia.

\section{AGRADECIMIENTOS}

Al Conselho Nacional de Desenvolvimento Científico e Tecnológico (CNPq) por el financiamiento al proyecto 303983/2014-0, Beca Productividad IA. A la revista Ciência \& Saúde Coletiva por el permiso para la utilización del artículo "A revista Ciência \& Saúde Coletiva e o processo de institucionalização de um campo de conhecimentos y práticas". A la Editora FIOCRUZ por el permiso para la utilización del Prefacio del libro Saúde Coletiva: a Abrasco em 35 anos de história. Al Espaço da Escrita de la Coordenadoria Geral da Universidade (CGU) de la Universidade Estadual de Campinas (Unicamp) por los servicios de traducción.

\section{REFERENCIAS BIBLIOGRÁFICAS}

1. Collyer F. Mapping the sociology of health and medicine: America, Britain and Australia compared. Houndmills, Basingstoke: Palgrave Macmillan; 2012.

2. Nunes ED. Prefácio. In: Lima NT, Santana JP, Paiva CHA. Saúde Coletiva: a Abrasco em 35 anos de história. Rio de Janeiro: Editora Fiocruz; 2015. p. 9-13.

3. Nunes ED. A revista Ciência \& Saúde Coletiva e o processo de institucionalização de um campo de conhecimentos e práticas. Ciência \& Saúde Coletiva. 2015;20(7):1975-1982. 
4. Nunes ED. A Saúde Coletiva e a educação médica. $53^{\circ}$ Congresso Brasileiro de Educação Médica; 7-10 nov 2015; Rio de Janeiro, Brasil.

5. Nunes ED. Saúde Coletiva: história de uma idéia e de um conceito. Saúde e Sociedade. 1994;3(2):5-21.

6. Nunes ED. Por una epistemología de la Salud Colectiva: los conceptos sociológicos. Salud Colectiva. 2010;6(1):11-19.

7. Paim JS, Almeida Filho N. Saúde coletiva: uma "nova" saúde pública ou campo aberto a novos paradigmas? Revista de Saúde Pública. 1998;32(4):299-316.

8. Luz MT. Complexidade do campo da Saúde Coletiva: multidisciplinaridade, interdisciplinaridade, e transdisciplinaridade de saberes e práticas análise sócio-histórica de uma trajetória paradigmática. Saúde e Sociedade. 2009;18(2):304-311.

9. Donnangelo MCF. A pesquisa em saúde coletiva no Brasil: a década de 70. In: Associaçäo Brasileira de Pós-Graduaçäo em Saúde Coletiva. Ensino da saúde pública, medicina preventiva e social no Brasil. Rio de Janeiro: ABRASCO; 1983. v. 2, p. 17-35.

10. Hochman G, Nunes ED. Abertura de uma nova seção na RC\&SC. Ciência \& Saúde Coletiva. 2015;20(1):137.

11. Pinto I, Paim J. A Abrasco e a experiência da graduação em Saúde Coletiva. In: Lima NT, Santana JP, Paiva CHA. Saúde Coletiva: a Abrasco em 35 anos de história. Rio de Janeiro: Editora Fiocruz; 2015. p. 137-150.

12. Nunes TCM, Fagundes TLQ, Soares CLM. Os cursos lato sensu na formação em Saúde Coletiva: evolução histórica e desafios contemporâneos. In: Lima NT, Santana JP, Paiva CHA. Saúde Coletiva: a Abrasco em 35 anos de história. Rio de Janeiro: Editora Fiocruz; 2015. p.151-167.

13. Dallegrave D, Ceccim RB. Residências em saúde: o que há nas produções de teses e dissertações. Interface-Comunicação, Saúde, Educação. 2013;17(47):759-776.

14. Barata RB. A Abrasco e a pós-graduação stricto sensu em Saúde Coletiva. In: Lima NT, Santana JP, Paiva CHA. Saúde Coletiva: a Abrasco em 35 anos de história. Rio de Janeiro: Editora Fiocruz; 2015. p. 169-198.

15. Belisário AS. Congressos da Abrasco: a expressão de um espaço consolidado. En: Lima NT, Santana JP, Paiva CHA. Saúde Coletiva: a Abrasco em 35 anos de história. Rio de Janeiro: Editora Fiocruz; 2015. p. 115-136.
16. Asnake M. A importância da publicação científica para o desenvolvimento da saúde pública. Ciência \& Saúde Coletiva. 2015;20(7):1972.

17. Nunes ED, Ferreto LE, Oliveira ALO, Nascimento JL, Barros NF, Castellanos MEP. O campo da Saúde Coletiva na perspectiva das disciplinas. Ciência \& Saúde Coletiva. 2010;15(4):1917-1922.

18. Carvalho YM, Ceccin RB. Formação e educação em saúde: aprendizados com a saúde coletiva. In: Campos GWS, Minayo MC, Akerman M, Drumond Júnior M, Carvalho YM. Tratado de Saúde Coletiva. Rio de Janeiro: Hucitec, Fiocruz; 2006. p. 149-182.

19. Souza LEPF. Saúde Pública ou Saúde Coletiva? Revista Espaço para a Saúde. 2014;15(4):1-21.

20. Minayo MCS. Global \& local, meritocrático \& social: o papel da ciência e tecnologia em Saúde Coletiva no Brasil. Tempus - Actas de Saúde Coletiva. 2012;6(2):11-26.

21. Vaitsman J, Ribeiro JM, Lobato LVC. Análise de políticas, políticas de saúde e a Saúde Coletiva. Physis - Revista de Saúde Coletiva. 2013;23(2): 589-611.

22. Almeida Filho N. Intersetorialidade, transdisciplinaridade e saúde coletiva: atualizando um debate em aberto. Revista de Administração Pública. 2000;34(6):11-34

23. Lima NT, Santana JP, (org.). Saúde Coletiva como compromisso: a trajetória da Abrasco. Rio de Janeiro: Editora Fiocruz; 2006.

24. Lima NT, Santana JP, Paiva CHA. Saúde Coletiva: a Abrasco em 35 anos de história. Rio de Janeiro: Editora Fiocruz; 2015.

25. Ribeiro PT. A instituição do campo científico da Saúde Coletiva no Brasil [Tese de Mestrado]. Rio de Janeiro: Escola Nacional de Saúde Pública, Fundação Oswaldo Cruz, Brasil; 1991.

26. García JC. La educación médica en América Latina. Washington: Organización Panamericana de la Salud; 1972.

27. Arouca ASS. O dilema preventivista: contribuição para a compreensão e crítica da medicina preventiva [Tese de doutorado]. Campinas: Unicamp; 1975.

28. Nunes ED. As ciências sociais em saúde na América Latina: tendências e perspectivas. Brasília: Organização Pan-Americana da Saúde; 1985. 
29. Paim JS. La salud colectiva y los desafíos de la práctica. In: Organización panamericana de la Salud. La crisis de la salud pública: reflexiones para el debate. Washington DC: OPS; 1992. p. 151-160.

30. Almeida-Filho N, (org.). Saúde coletiva: teoria e prática. Rio de Janeiro: MedBook; 2014.

31. Ben David J. The scientific role: the conditions of its establishment. Minerva. 1965;4(1):15-54.

32. Oberschall A, (ed.). The establishment of empirical sociology. New York: Harper and Row; 1972.

33. Bloom SW. The word as a scapel: A history of medical sociology. New York: Oxford University Press; 2002.

34. Leavell H, Clark EG. Medicina Preventiva. São Paulo: McGraw-Hill; 1976.

35. García JC. Paradigmas para la enseñanza de las ciencias sociales en las escuelas de medicina. Educación Médica y Salud. 1971;5(2):130-148.

36. OPS/OMS. Aspectos teóricos de las Ciencias Sociales aplicadas a la medicina. Educación Médica y Salud. 1974;8(4):354-359.

37. Gandra Júnior DS. A lepra: uma introdução ao estudo do fenômeno social da estigmatização [Tese de doutorado]. Belo Horizonte: Universidade Federal de Minas Gerais; 1970.

38. Donnangelo MCF. Medicina e sociedade: o médico e seu mercado de trabalho. São Paulo: Pioneira; 1975.

39. Donnangelo MCF, Pereira L. Saúde e sociedade. São Paulo: Duas Cidades; 1976.

40. Machado R, Loureiro A, Luz R, Muricy K. Danação da norma: a medicina social e constituição da psiquiatria no Brasil. Rio de Janeiro: Graal; 1978.

41. Luz MT. As instituições médicas no Brasil. Rio de Janeiro: Graal; 1979.

42. Cordeiro H. A indústria da saúde no Brasil. Rio de Janeiro: Graal; 1980.

43. Cohn A. Previdência social e populismo. [Tese de doutorado]. São Paulo: Universidade de São Paulo; 1980.

44. Schraiber LB. Educação médica e capitalismo. São Paulo: Hucitec; 1989.

45. Marsiglia RMG. Assistência médica e relações de trabalho na empresa: O modelo de convênios com a Previdência Social [Tese de mestrado]. São Paulo: Universidade de São Paulo; 1981.

46. Teixeira SMF. Assistência médica na previdência social: evolução e crise de uma política social. [Tese de mestrado]. Rio de Janeiro: Instituto Universitário de Pesquisas do Rio de Janeiro; 1976.

47. Mendes-Gonçalves RB. Medicina e História: raízes sociais do trabalho médico. [Tese de mestrado]. São Paulo: Faculdade de Medicina da Universidade de São Paulo; 1979.

48. Paim JS. Medicina comunitária: introdução a uma análise crítica. Saúde Debate 1976;(1):9-11.

49. Braga JCS. A questão da saúde no Brasil : um estudo das politicas sociais em saúde publica e medicina previdenciária no desenvolvimento capitalista. [Dissertação de Mestrado]. Campinas: Instituto de Economia, Universidade Estadual de Campinas; 1978

50. Caldas Jr. L. Epidemiologia e controle da doença de Chagas: relação com a estrutura agrária na região de Sorocaba. [Tese de mestrado]. São Paulo: Faculdade de Medicina, Universidade de São Paulo; 1980.

51. Goldbaum M. Doença de Chagas e trabalho em Área urbana. São Paulo: Faculdade de Medicina, Universidade de São Paulo; 1979

52. Silva LJ. A evolução da doença de Chagas no Estado de São Paulo. [Tese de doutorado]. Ribeirão Preto: Faculdade de Medicina de Ribeirão Preto, Universidade de São Paulo; 1981.

53. Breilh J. Economia, medicina, política. Quito: Editora Universitaria; 1979.

54. Laurell AC. El estudio social del proceso salud-enfermedad en América Latina. Cuadernos Médico Sociales.1986;(17):3-18.

55. Iriart C, Waitzkin H, Breilh J, Estrada A, Merhy, EE. Medicina social latinoamericana: aportes y desafíos. Revista Panamericana de Salud Pública. 2002; 12(2):128-136.

56. Organización Panamericana de Salud. Informe del Comité del Programa de Libros de Texto de la OPS para la Enseñanza de la Medicina Preventiva y Social en las Escuelas de Medicina de la América Latina [Internet]. 1974 (Serie de Desarrollo de Recursos Humanos No 2) [citado 15 ene 2016]. Disponible en: http://goo.gl/5PlihJ.

57. Bojalil LF, García JC. La Universidad Autónoma Metropolitana-Xochimilco: consideraciones sobre 
el marco teórico de una práctica universitaria. México: UAM; 1981.

58. Escorel S. Reviravolta na saúde: origem e articulação do movimento sanitário. Rio de Janeiro: Editora Fiocruz; 1999.

59. Giovanella L. As origens e as correntes atuais do enfoque estratégico em planejamento de saúde na América Latina. Cadernos de Saúde Pública. 1991;7(1):26-44.

60. CEBES. A questão democrática na área de Saúde. Saúde em Debate. 1980;(9):11-13.

61. Tambellini AT, Botazzo C, Nunes GC, Buss P. A Abrasco e os anos de chumbo: a Comissão da Verdade no campo da saúde. In: Lima NT, Santana JP, Paiva CHA. Saúde Coletiva: a Abrasco em 35 anos de história. Rio de Janeiro: Editora Fiocruz; 2015. p. 69-99.

62. Associação Brasileira de Pós-Graduação em Saúde Coletiva. Ensino da Saúde Pública, Medicina Preventiva e Social no Brasil: Relatório Final. Rio de Janeiro: ABRASCO; 1982.

63. Ferreira LO. Os periódicos médicos e a invenção de uma agenda sanitária para o Brasil (1827-43). História, Ciências, Saúde-Manguinhos. 1999;6 (2):331-351.

64. Minayo MCS, Gomes R, Almeida MF, Goldbaum M, Carvalhiero JR. A Abrasco faz ciência e avança em conhecimentos: contribuições da Ciência \& Saúde Coletiva e da Revista Brasileira de Epidemiologia. In: Lima NT, Santana JP de, Paiva CHA. Saúde Coletiva: a Abrasco em 35 anos de história. Rio de Janeiro: Editora Fiocruz; 2015. p. 101-114.
65. Campos GWS, et al. Tratado de Saúde Coletiva. 2a ed. São Paulo: Hucitec; 2012.

66. Silva J, Almeida-Filho N. Saúde Coletiva: teoria e prática. Rio de Janeiro: MedBook; 2014.

67. Hughes EC. Men and their work. Glencoe: Free Press; 1958.

68. Conselho Nacional de Desenvolvimento Científico e Tecnológico. Diretório dos grupos de pesquisa no Brasil: Lattes [Internet]. Brasília: CNPq [citado 15 ene 2016]. Disponible en: http://goo. $\mathrm{gl} / \mathrm{Zrp} 3 \mathrm{fv}$.

69. Cesse EAP, Veras MASM. A Abrasco e a experiência dos mestrados profissionais. In: Lima NT, Santana JP, Paiva CHA. Saúde Coletiva: a Abrasco em 35 anos de história. Rio de Janeiro: Editora Fiocruz; 2015. p. 199-213.

\section{NOTAS FINALES}

[a] En la sección "Construtores da Saúde Coletiva" de la revista Ciência \& Saúde Coletiva, durante el año 2015, se publicaron 12 trabajos que abordan la vida profesional de Juan César García, Samuel Barnsley Pessoa, Ricardo Bruno Mendes Gonçalves, Rodolfo dos Santos Mascarenhas, Maria Cecilia Ferro Donnangelo, Izabel dos Santos, Guilherme Rodrigues da Silva, Mário Magalhães, Walter Leser, Joaquim Alberto Cardoso de Melo, Giovanni Berlinguer y Maria Cecília Puntel de Almeida.

[b] Respecto a las revistas brasileñas, consultar el número temático "A importância das revistas de Saúde Pública/Saúde Coletiva para o SUS e para a ciência brasileira" publicado por la revista Ciência \& Saúde Coletiva, en el volumen 20, número 7 de 2015.

\section{FORMA DE CITAR}

Nunes ED. La salud colectiva en Brasil: analizando el proceso de institucionalización. Salud Colectiva. 2016;12(3):347360. doi: $10.18294 /$ sc.2016.894.

Recibido: 18 de marzo de 2016 | Versión final: 17 de mayo de 2016 | Aprobado: 22 de junio de 2016

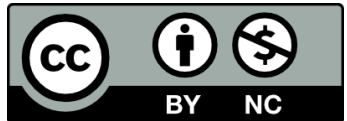

Este obra está bajo una licencia de Creative Commons Reconocimiento-NoComercial 4.0 Internacional. Reconocimiento - Permite copiar, distribuir y comunicar públicamente la obra. A cambio, se debe reconocer y citar al autor original. No Comercial - Esta obra no puede ser utilizada con finalidades comerciales, a menos que se obtenga el permiso.

http://dx.doi.org/10.18294/sc.2016.894 